\title{
Do N-planet systems have a boundary between chaotic and regular motions?
}

\author{
Ji-Lin Zhou, Yi-Sui SUN \\ Department of Astronomy, Nanjing University, Nanjing 210093, China; zhoujl@nju.edu.cn
}

\begin{abstract}
Planetary systems consisting of one star and $n$ planets with equal planet masses $\mu$ and scaled orbital separation are referred as EMS systems. They represent an ideal model for planetary systems during the post-oligarchic evolution. Through the calculation of Lyapunov exponents, we study the boundary between chaotic and regular regions of EMS systems. We find that for $n \geq 3$, there does not exist a transition region in the initial separation space, whereas for $n=2$, a clear borderline occurs with relative separation $\sim \mu^{2 / 7}$ due to overlap of resonances (Wisdom, 1980). This phenomenon is caused by the slow diffusion of velocity dispersion ( $\sim t^{1 / 2}, t$ is the time) in planetary systems with $n \geq 3$, which leads to chaotic motions at the time of roughly two orders of magnitude before the orbital crossing occurs. This result does not conflict with the existence of transition boundary in the full phase space of N-body systems.
\end{abstract}

Subject headings: N-body Problem; Stability; Chaos; Diffusion.

\section{Introduction}

Planetary system is a paradigm of classical N-body problems. The pioneer work of Poincaré(1) indicated that a general N-body system with $N \geq 3$ is not integrable. According to the work of Arnold(2), a typical near-integrable Hamiltonian system with more than 3 degrees of freedom is topologically unstable, i.e, the action variables can diffuse away in the phase space during a sufficient long period. However, for a specific planetary system, when the instability is significant and how long the system can be stable are unsolved problems with great astronomical interests( $(\underline{3})$.

One of the most interesting questions is whether there exists a transition region between

regular and chaotic motions in a $n$-planet system. Gladman(4) found that for $n=2$, the orbits of two planets with equal masses $(\mu)$ will be chaotic as long as their relative separation $\geq 2 \mu^{2 / 7}$. The scaling $\mu^{2 / 7}$ was first found by Wisdom(5) in a restricted three-body model 
with a resonance overlap criterion. However, whether there exist similar relationships for systems with $n \geq 3$ remains unknown.

In a previous paper $((\underline{6})$, hereafter Paper I), we investigated the orbital crossing time and orbital diffusion of planetary systems with equal planet masses and scaled separation (we call them the EMS systems). We found that for EMS systems with $n \geq 3$, the velocity dispersion of the planets diffuses with time $t$ as $\sim t^{1 / 2}$. In this paper, we study the transition from regular to chaotic regions in the initial separation space of these systems.

\section{Model and Method}

An EMS system consists of a star with unit mass $\left(\mu_{0}=1\right)$ and $n$ planets with equal masses $\mu(\mu \ll 1)$ moving around it. Assume all the planets are initially in circular and coplanar orbits with semi-major axes $a_{i}$, where $i=1, \ldots, n$ are sequenced from inner to outside. We put the innermost planet at $a_{1}=1 \mathrm{AU}$, which is the average distance from the Sun to the Earth. The initial separation between the planetary orbits, denoted as $k_{0}$, are equal when scaled by their mutual Hill's radii $R_{H}$,

$$
k_{0}=\frac{a_{i+1}-a_{i}}{R_{H}}, \quad R_{H}=\left(\frac{2 \mu}{3}\right)^{1 / 3} \frac{a_{i}+a_{i+1}}{2}, \quad(i=1, \ldots, n-1) .
$$

The initial mean anomaly $M_{i}$ and longitude of perihelion $\varpi_{i}$ of each planet orbit are chosen randomly.

In an inertial coordinate with origin on the mass center of the $n+1$ bodies ( the star and $n$ planets), the Hamiltonian of the system is given as,

$$
H=\sum_{i=0}^{n} \frac{\mathbf{p}_{i}^{2}}{2 \mu_{i}}-\sum_{i=0}^{n} \sum_{j=0, j>i}^{n} \frac{G \mu_{i} \mu_{j}}{r_{i j}}
$$

where $\mathbf{r}_{i}$ and $\mathbf{p}_{\mathbf{i}}=\mu_{i} \dot{\mathbf{r}}_{i}(i=0, \ldots n)$ are the position and momentum of the star and the planets, respectively, $r_{i j}=\left|\mathbf{r}_{j}-\mathbf{r}_{i}\right|$ is the distance between bodies $i$ and $j, \mathrm{G}$ is the gravitational constant.

The equations of motion corresponding to eq. (2) are integrated by a Runge-Kutta method with adaptive step-sizes. The orbits are simulated up to $10^{7}$ years, with an accumulated error of relative energy $\sim 10^{-8}$ for weak chaotic or regular orbits and $\sim 10^{-6}$ for strong chaotic orbits in the case of $n=3$ (Fig.1a). The Lyapunov exponents are defined as the limit of $\chi(t)$ when time $t$ tends to infinity, with

$$
\chi(t)=\frac{1}{t} \log \left(\frac{|\xi(t)|}{|\xi(0)|}\right),
$$



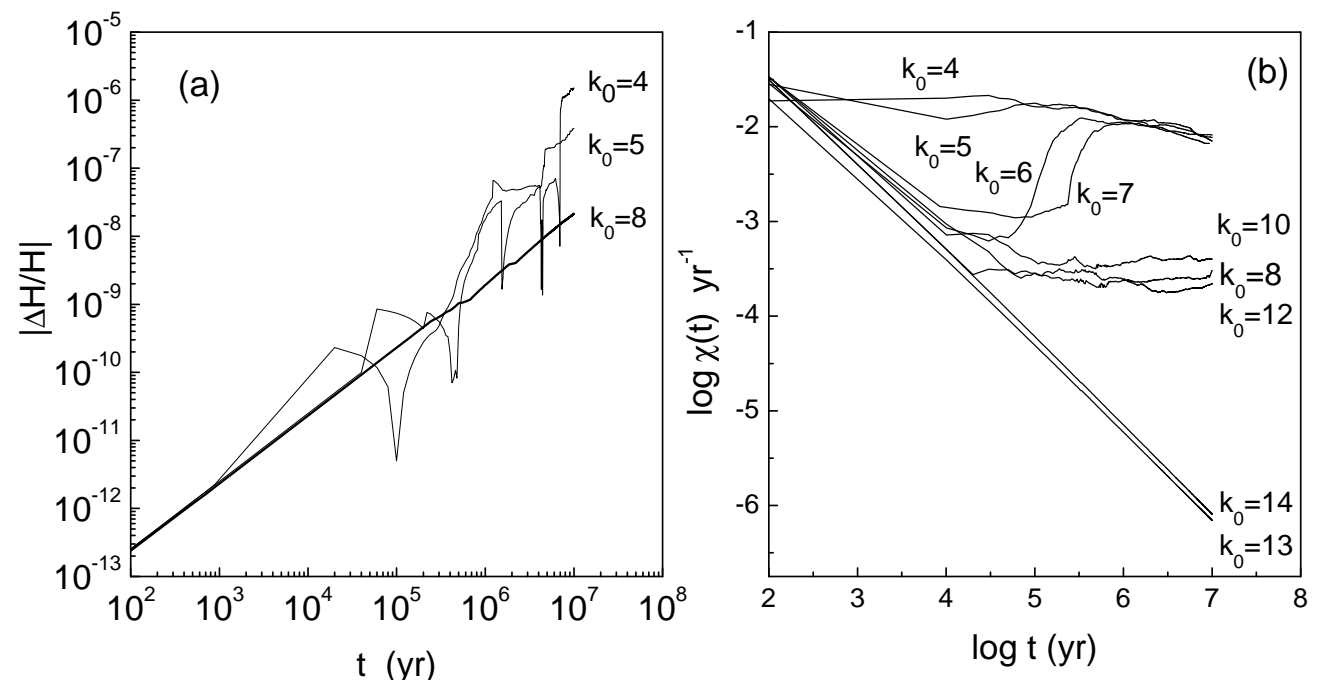

Fig. 1. - Evolution of 3-plant EMS systems with mass $\mu=10^{-8}$. (a) Variations of accumulated error of the relative energy with time. The three lines correspond to initial separation $k_{0}=4,5,8$, respectively. (b) Variations of $\chi(t)$ for orbits with different initial separation $k_{0}$.

where $\xi(t)$ and $\xi(0)$ are tangential vectors in the phase space at time $t$ and 0 , respectively, $|\cdot|$ is the norm of a vector. We solve the variational equations of eq. (2) along the solution to get $\chi(t)$. Normalization of $\xi(t)$ at every fixed time interval is required to avoid overflow during the simulations.

\section{Numerical Results}

A notable phenomenon in an EMS system with $n \geq 3$ is for some orbits, $\xi(t)$ may reach a limit of medium value $\left(\sim 10^{-3} \mathrm{yr}^{-1}\right.$ ) at time $t \sim 10^{5} \mathrm{yrs}$, then it grows to a large limit ( $\left.\sim 10^{-2} \mathrm{yr}^{-1}\right)$ after a long period time $\left(\sim 10^{7} \mathrm{yrs}\right)$, indicating that the orbits finally become quite chaotic after a long time in a transitional state (e.g., orbits with $k_{0}=6,7$ in Fig.1b). Moreover, we find that these transition orbits are in the border region between strong (with large Laypunov exponents) and weak (with medium ones) chaotic motions.

The transition phenomenon of $\chi(t)$ does not occur in EMS systems with $n=2$. As shown in Fig.2a, different integration time spans give almost identical border $k_{c}$ between chaotic and regular motions. For $\mu=10^{-8}, k_{c} \approx 5.2$, which roughly coincides with the location predicted by Wisdom and Gladman's criterion, i.e., $k_{c}=2 \mu^{2 / 7} /(2 \mu / 3)^{1 / 3} \approx 2.3 \mu^{-1 / 21} \approx 5.5$.

The evolution of $\chi(t)$ is linked with the velocity dispersion $(\sigma)$ in the planetary systems. As demonstrated in paper I, one of the major characteristics of planetary systems with $n \geq 3$ is that $\sigma$ diffuses slowly with $\sim t^{1 / 2}$ due to the interactions between planets. The growth of velocity dispersion (orbital eccentricities) of the planets enhances the planetary 

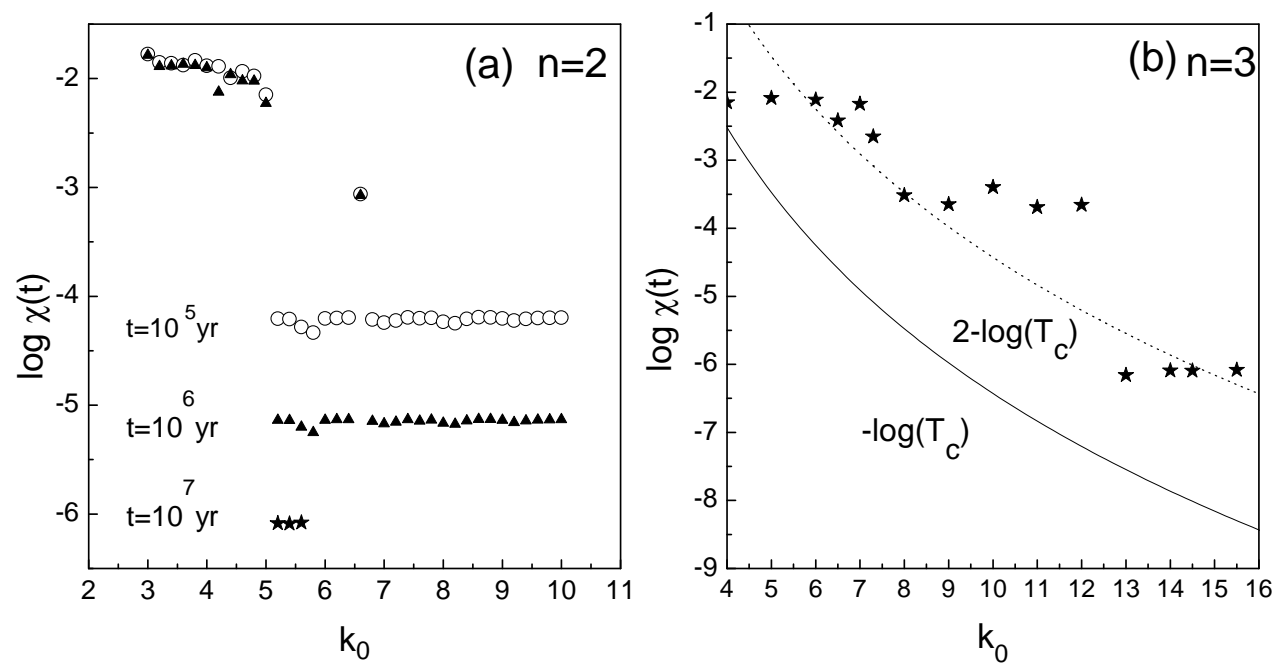

Fig. 2.- (a) Variations of $\chi(t)$ with $k_{0}$ at different time: $10^{5} \mathrm{yr}$ (circles), $10^{6} \mathrm{yr}$ (triangles) and $10^{7} \mathrm{yr}$ (stars) in a 2-planet EMS system with mass $\mu=10^{-8}$. (b)Variations of $\chi(t)$ with $k_{0}$ at $t=10^{7} \mathrm{yr}$ (stars) in a 3-planet EMS system with mass $\mu=10^{-8}$. The solid and dotted curves plot the functions $-\log \left(T_{c}\right)$ and $2-\log \left(T_{c}\right)$, respectively.

perturbations, leads to the growth of $\chi(t)$ after a transition stage, and finally orbital crossing may occur after the systems undergo a long period of strong chaotic motions. Define the orbital crossing time $T_{c}$ as the minimum duration that either orbit crossing ( $a_{i} \geq a_{j}$ for $i<j)$ or close encounter $\left(r_{i j}<R_{H}\right)$ occurs. In paper I, we derived an empirical formula of $T_{c}$ for $k_{0}>2.3,10^{-4} \leq \mu \leq 10^{-10}$. For planets in circular orbits, it gives,

$$
\log \left(\frac{T_{\mathrm{c}}}{\mathrm{yr}}\right)=(-2-0.27 \log \mu)+(18.7+1.1 \log \mu) \log \left(\frac{k_{0}}{2.3}\right) .
$$

Fig.2b plots the $\chi\left(10^{7} \mathrm{yr}\right)$ and quantities $-\log \left(T_{c}\right)$. A relationship $\log \left(\chi\left(10^{7} \mathrm{yr}\right)\right) \sim 2-\log \left(T_{c}\right)$ suggests that the time scale that an orbital becomes chaotic (i.e. Lyapunov time) is roughly two orders of magnitude less than the orbital crossing time. The correlation indicates that the growth of $\chi(t)$ is due to the slow orbital diffusion towards high velocity dispersion. Since growth of velocity dispersion is a robust procedure in a planetary system with $n \geq 3$, we believe that the transitional regular orbits will eventually become chaotic after sufficient long time, hence there is no clear boundary between the domains of chaotic and regular motions.

\section{Conclusion}

In this paper, we study the boundary between chaotic and regular motions in EMS systems with $n$ planets. Our results indicate that the growth of velocity dispersion is robust as long as $n \geq 3$, which gradually turns the orbits initially in regular regions to chaotic 
ones. Hence there does not exist a borderline in the initial separation space, which does not conflict with the existence of boundaries in the full phase space (position and velocity space) of N-body systems.

\section{Acknowledgements}

We thanks Prof. D.N.C. Lin for helpful discussions. This work is supported by NSFC (No.10233020,10778603) and NCET (04-0468) of China.

\section{REFERENCES}

H. Poincaré,, Les Méthodes Nouvelles de la Mécanique Céleste (Gauthier-Villars, Paris, 1892)

V. I. Arnold, Dokl. Akad. Nauk SSSR 156, 9 (1964)

J. Laskar, Nature 338, 237 (1989)

B. Gladman, Icarus 106, 247 (1993)

J. Wisdom, AJ 85,1122 (1980)

J.L. Zhou, D.N.C. Lin, \& Y.S. Sun, Post-Oligarchic Evolution of Protoplanetary Embryos and the Stability of Planetary Systems, accepted by ApJ, arXiv:astro-ph/0705.2164, (2007), (Paper I) 